\title{
Memory processes in facial recognition and recall
}

\author{
MICHELLE E. COHEN and CALVIN F. NODINE \\ Department of General Educational Psychology, Temple University, Philadelphia, Pennsylvania 19122
}

\begin{abstract}
The encoding processes of recognition and recall for line-drawn faces were investigated. Subjects randomly received three-alternative forced-choice tests of recognition and probe recall of 20 male faces. Between each inspection and test, subjects performed an interference task for $10 \mathrm{sec}$. The interference tasks consisted of either identifying the missing facial feature in line drawings or in photographs, or correctly identifying the misspelled words describing different facial features. The results indicate that recognition performance was higher than proberecall performance for all groups. The analysis of the recognition data suggests that recognition ability decreased as the similarity of the interference task to the target increased. This finding suggests that faces are encoded using visual rather than verbal imagery.
\end{abstract}

How do we remember the faces of people to whom we have just been introduced? How do we recognize a face in a crowd or describe the face of an acquaintance? Often, memory of another is mediated through visual perception of the facial configuration. The processes used to perceive and remember faces are specific aspects of the processing of visual stimuli. Studies of the human face as a complex visual stimulus have shown that man is well adapted to perceive and remember many different faces (Friedman \& Haber, 1974; Friedman, Reed, \& Carterette, 1971; Howells, 1938). Friedman and Haber (1974) have proposed that the reason faces are rarely forgotten is that the individual perceiving a face employs some process of organization to encode that particular face in memory.

The above studies support the notion that memory for a face is dependent upon the processing of the facial configuration. The question arises as to how these facial features are encoded into memory. The linguistic approach has been hypothesized to describe a process of analyzing a visual pattern into a hierarchy of verbal descriptions (Reed \& Angaran, 1972). This verbal encoding process has been supported by Bower and Karlin (1974) in a study of depth of processing and facial recognition.

Studies concerned with visual stimuli other than faces support a dual coding hypothesis, similar to that proposed by Paivio's (1971) work in visual imagery. den Heyer and Barrett (1971) investigated the encoding of visual and verbal information in short-term memory. By means of visual and verbal interpolated tasks, they found evidence for dual modalities in short-term memory. Information pertaining to a feature within the stimulus was found to be mediated by a visual code, while identity information used both visual and verbal codes. A later study by Reed and Johnson (1975) suggested a dual encoding process that was more

Part of this paper was presented at the Eastern Psychological Association meeting in Washington, D.C., 1978. strongly affected by verbal concepts. They hypothesized that visual imagery has a limited capacity in working memory and is more prone to interference. Therefore, long-term retention of verbal concepts is needed to enhance the visual code. Bencomo and Daniel (1975) and Pellegrino, Siegel, and Dhawan (1975) concluded that pictures are encoded in terms of both imaginal and semantic features. Reed (1972) hypothesized that subjects used an "abstract image or prototype" to describe categories of facial features. New faces are then encoded on the basis of their similarity to these categories.

Bradshaw and Wallace (1971) have suggested that in the process of facial recognition, individuals may employ a "rapid sequential self-terminating scan" of the features in the facial configuration. Smith and Nielson (1970) investigated the effects of recognition and recall procedures on short-term memory for schematic faces. They proposed that different processes were acting in the two tasks. Tests of recognition appeared to employ a sequential scanning of the stimulus, starting at the top and scanning to the bottom of the figure; whereas probe recall $1 \mathrm{sec}$ after presentation produced retrieval as a unit, but after $10 \mathrm{sec}$ there was evidence of verbal encoding of a feature list.

In consideration of the findings presented above, the purpose of this study was to investigate the encoding procedures involved in the recall and recognition of faces. This purpose was explored through the use of visual and verbal interpolated tasks.

\section{METHOD}

\section{Subjects}

Twelve graduate students and faculty members served as judges for the initial phase of this study. Fifty-four undergraduate students served as subjects in the experimental manipulation.

\section{Apparatus}

The faces used in this study were line drawings of male faces constructed from the Identi-Kit (Smith-Wesson law enforcement 
group) series of facial-feature overlays; a procedure often used by law enforcement agencies. Twenty target faces were used. Fifteen different faces (line drawings) were constructed for the visual interpolated tasks. Each of the interpolated task faces were used in five different displays; each display differed by the elimination of one of the seven primary features (eyebrows, eyes, ears, nose, mouth, chin, or hair). The distractor faces used in the recognition tests were line drawings that varied from the target face by two, four, or all seven facial features. The photographs used in this study were 80 black-and white portrait photographs of males between the ages of 25 and 50 years. The photographs were taken from journal and magazine advertisements that were composed of uniform pictures of male executives (e.g., insurance advertisements).

\section{Procedure}

Prior to the testing of treatment groups, 12 judges were shown the 20 target faces one at a time and were asked to assign one of three size dimensions (small, medium, or large) to the five basic features of each face (eyebrows, eyes, ears, nose, and lips). The size dimension assigned to each feature was to be relative to that particular facial configuration in which it was contained. These dimensions were used to assess the subjects' answers in the probe-recall portions of the experiment.

Intact classroom groups were randomly assigned to one of three treatments (Group 1, $N=14$; Group 2, $N=12$; Group 3, $\mathrm{N}=28$ ). Treatment groups differed in procedure according to the type of interpolated task they received. Group 1 viewed 20 line-drawn faces during each interpolated task interval. Each face had one facial feature missing; subjects were to identify the missing feature. Group 2 received a similar task, with the exception being that the 20 faces were photographic portraits of male faces. Group 3 was presented with a series of 20 misspelled words each naming a different facial feature. The subjects in this group were asked to identify the word and the misspelling. All subjects' responses were self-recorded on individual answer sheets.

Each group received two training trials (one recognition and one recall) and 20 experimental trials (10 recognition and 10 recall randomly distributed in each treatment group). All stimuli were presented on $16-\mathrm{mm}$ film to control the presentation times. Each trial followed the format shown in Figure 1: 2-sec inspection of the line-drawn male target face, 10-sec interpolated interval, and 4-sec test period. Following each interpolated task interval, a word appeared on the screen that signified the type of test the subjects would receive. Subjects were not aware of the type of memory test they would receive until the signal was flashed on the screen. The word "recognition" signified that the subjects would be asked to identify the target face within a three-alternative forced-choice test and assign a confidence rating to their choice. The word "recall" flashed on the screen signified that subjects would be participating in a probe-recall procedure. The probe-recall test consisted of the presentation of a word representing one of the five facial features used to compose the target face (eyebrows, eyes, ears, nose, and lips) and a three-dimension size scale (small, medium,

$$
\text { TARGET } \sqrt{2 \text { Sec. }}
$$

TASK

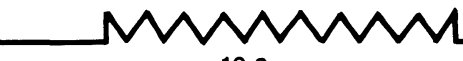

$10 \mathrm{Sec}$.

TEST

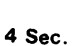

Figure 1. Testing procedures for all groups.

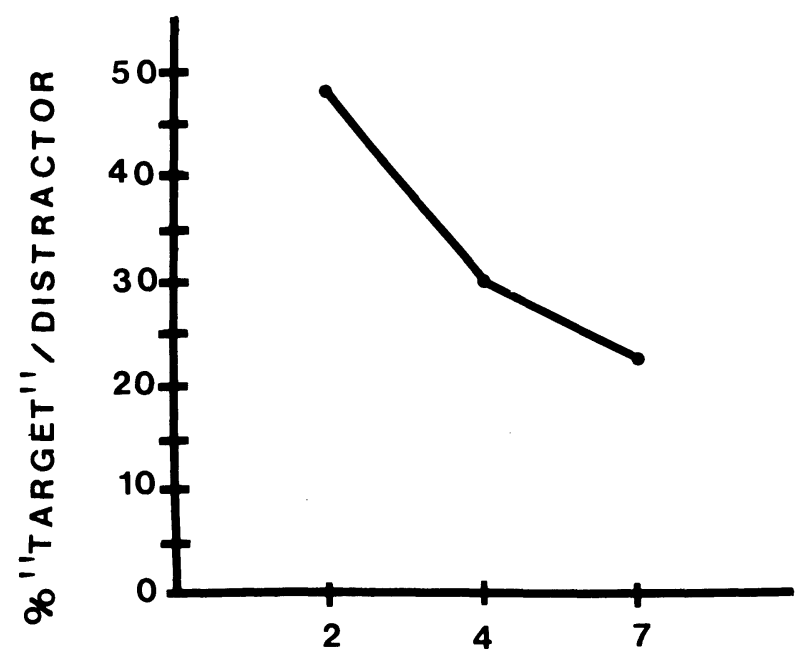

\section{DEGREE OF DISSIMILARITY}

Figure 2. Percentage of distractors chosen as target.

and large) randomly ordered below the probed feature. The subjects' task was to assign the appropriate size dimension to the probed feature as they remembered its appearance in the target face and assign a confidence rating to their choice.

\section{RESULTS}

A 3 (interpolated task) by 2 (memory test) fixed effects analysis of variance with repeated measures on the second factor was performed on the subjects' correct response data. The main effect for test was significant $[F(1,51)=150.6, p<.01]$, as was the Task by Test interaction $[F(2,51)=4.13, p<.05]$. The post hoc test of pairwise comparisons showed that subjects did significantly better on tests of recognition than on tests of recall in all three treatment groups [Group 1, $\mathrm{F}(1,13)=14.34, \mathrm{p}<.01$; Group 2, $\mathrm{F}(1,11)=46.49$, $\mathrm{p}<.01$; Group 3, $\mathrm{F}(1,27)=98.03, \mathrm{p}<.01]$. The test for simple main effects of the Task by Test interaction was significant for recognition tests $[F(2,53)=3.21$, $\mathrm{p}<.05$ ] but not for recall tests. Post hoc $t$ tests between the treatment groups on tests of recognition showed that the group receiving the word interpolated task (Group 3) did significantly better on recognition tests than the group receiving the line-drawn task (Group 1) $\left[\mathrm{t}_{1,3}(40)=2.68, \mathrm{p}<.01\right]$. The remaining pairwise comparisons showed no significant differences between the groups on tests of recognition.

Figure 2 shows the percentage of distractor faces accepted as targets on recognition tests. Analysis of the target and distractor data showed that target faces yielded more true positive responses $(80.4 \%$ hits) than did the distractors ( $19.6 \%$ false positives). The falsepositive responses (misses) were in the expected direction, as shown in Figure 2. Distractors with two 
features different from the target faces were most often chosen as the target, followed by distractors with four different features, while distractors with seven (all) different features were chosen as the target least of all. These results were significantly different from the expected response frequencies $\left[\chi^{2}(2)=6.31, p<.05\right]$. The target faces yielded few false-negative responses (9.8\%), while distractors contributed more rejections (90.2\%).

Analysis of subjects' confidence ratings for their choice of target faces on recognition produced results consistent with the findings presented above. Subjects in Group 3 (word interpolated task) had more confidence in their choice of target faces than subjects in other groups (in Group 3, 92.7\% were very confident in their choice of target faces; in Group 2, the photographic interpolated task, $90.2 \%$ were very confident in their choice; in Group 1, the line-drawn interpolated task, $85.5 \%$ were very confident in their choice). Even when subjects had no confidence in their choices and were considered to be guessing, subjects in Group 3 correctly identified considerably more target faces than subjects in Group 1 (Group 3, 60\% correct when guessing; Group 1, 52\% correct).

\section{DISCUSSION}

The results of this study suggest that the process of facial recognition is primarily dependent on a stored visual image. The closer the interpolated task was to the target, the more it interfered with the subject's recognition ability. If a verbal list had been used in the encoding process, then the word list task would have been expected to produce more interference with memory than it did. Further support of this interpretation comes from the effect of the distractors on recognition ability. As the distracting faces in the recognition triad came closer to the target faces in the number of facial features that were the same, distractors were more likely to be chosen as the target face. This suggests that the encoding process may depend on extraction of a prototype.

If some prototypical process was used in short-term storage of the faces, then the closer the distractors were to the target face, the more likely they were to fall within the realm of the prototype (or central tendency) for the features in that target face, and therefore, be accepted as the target. Because a prototype is likely to follow the central tendencies of the distinctive features within a facial configuration, it may be easier for subjects to process and use a prototype in recognition than a verbal list or description. Since inspection of a given target face was relatively brief in this study, subjects may have found the processes used in developing a prototype to be the most efficient means of processing and storing the stimuli. When distractors varied greatly from the target, and therefore varied greatly from the prototype, subjects had little difficulty in rejecting them.

The prototype of the target face may have failed to provide sufficient information for probe recall, resulting in the poor recall performance in this study. Probe recall in this study required the fine discrimination of size differences of facial features. Perhaps the storage of a prototype did not allow for this discrimination. Recall may need more processing time than was possible in the short time allotted in this study. Further investigation is needed to determine why recognition appeared to be easier in this particular design. The area of facial recall still presents many questions as to the exact nature of the process and its specific encoding and retrieval demands.

\section{REFERENCES}

Bencomo, A. A., \& Daniel, T. C. Recognition latency for pictures and words as a function of encoded-feature similarity. Journal of Experimental Psychology: Human Learning and Memory, 1975, 104, 119-125.

Bower, G. H., \& KARLIN, M. B. Depth of processing of faces and recognition memory. Journal of Experimental Psychology, 1974, 103, 751-757.

Bradshaw, J. L., \& Wallace, G. Models of processing and identification of faces. Perception \& Psychophysics, 1971, 9, 443-448.

DEN HEYER, K., \& BARRett, B. Selective loss of visual and verbal information in STM by means of visual and verbal interpolated tasks. Psychonomic Science, 1971, 25, 100-102.

Friedman, J., \& HABER, R. N. One reason why we rarely forget a face. Bulletin of the Psychonomic Society, 1974, 3, 107-109.

Friedman, M. P., Reed, S. K., \& Carterette, E. C. Feature saliency and the recognition memory for schematic faces. Perception \& Psychophysics, 1971, 10, 47-50.

Howells, T. H. The study of the ability to recognize faces. Journal of Abnormal and Social Psychology, 1938, 33, 124-127.

PaIvio, A. Imagery and verbal processes. New York: Holt, Rinehart, \& Winston, 1971.

Pellegrino, J. W., Siegel, A. W., \& Dhawan, M. Shortterm retention of pictures and words: Evidence for dual coding systems. Journal of Experimental Psychology: Human Learning and Memory, 1975, 104, 95-102.

ReEd, S. K. Pattern recognition and categorization. Cognitive Psychology, 1972, 3, 383-407.

Reed, S. K., \& Angaran, A. J. Structural models and embedded-figure difficulty for normal and retarded children. Perceptual and Motor Skills, 1972, 35, 155-164.

REed, S. K., \& Johnson, J. A. Detection of parts in patterns and images. Memory \& Cognition, 1975, 3, 655-673.

Smith, E. E., \& Nielsen, G. D. Representation and retrieval processes in short-term memory: Recognition and recall of faces. Journal of Experimental Psychology, 1970, 85, 397-405.

(Received for publication July 10, 1978.) 